\title{
Empreendedorismo Social e Inovação Social: enfoque, finalidades e conexões conceituais
}

\author{
Social Entrepreneurship and Social Innovation: focus, purposes, and conceptual connections
}

\section{Luiz Cláudio Ribeiro Machado}

Como citar esse artigo. Machado, L.C.R.. Empreendedorismo Social e Inovação Social: enfoque, finalidades e conexões conceituais. Revista Mosaico, v.11, n.2, p. 35-46, 2020.

Nota da Editora. Os artigos publicados na Revista Mosaico são de responsabilidade de seus autores. As informações neles contidas, bem como as opiniões emitidas, não representam pontos de vista da Universidade de Vassouras ou de suas Revistas.

\section{Resumo}

O objetivo deste trabalho é discutir as conexões conceituais entre construtos na adoção de uma perspectiva empreendedora com foco em inovação social. Há um posicionamento comum entre pesquisadores de que quando se descreve o empreendedorismo social (ES) e a inovação social (IS) muitos consideram estes termos sinônimos; entretanto, neste artigo, eles serão tratados como aspectos distintos de uma ação maior para um bem comum. Os procedimentos metodológicos seguiram para uma revisão sistemática qualitativa, utilizando a análise de conteúdo para extrair temas dos textos que tratam tanto de inovação social como empreendedorismo social. Dentre os aspectos que encontramos como maiores aproximações entre ES e IS são, em suma, que: a) a IS precisa de um meio social para se concretizar, assim como o ES; b) alguns tipos de ES podem gerar IS; c) o surgimento de ES e IS independem de setores específicos; d) ES e IS trazem mudanças nas práticas sociais; e e) tipos de ES e IS podem conviver conjuntamente em um ambiente de rede. Concluiu-se que, com a inovação social sendo também causada pelo empreendedorismo, algumas implicações podem ser observadas quando se trata da gestão social, principalmente no que concerne a participação social nas mudanças mais sistêmicas. Recomenda-se que nos estudos de ES e IS estejam manifestadas em seus textos quais são os paradigmas que estão sendo alicerçados, assim como posições filosóficas ou alegações do conhecimento de forma que seja possível o delineamento de um caminho epistemológico mais claro. .

Palavras-chave: Empreendedorismo Social; Inovação Social; Conexões conceituais.

\begin{abstract}
The aim of this paper is to discuss the conceptual connections between constructs in the adoption of an entrepreneurial perspective with a focus on social innovation. There is a common position among researchers that when describing social entrepreneurship (ES) and social innovation (IS) many consider these terms synonymous, however in this article, they will be treated as distinct aspects of an action greater for a common good. The methodological procedures followed for a systematic qualitative review using content analysis to extract themes from the texts that deal with both social innovation and social entrepreneurship. Among the aspects that we find as the closest approximations between ES and IS are, in short, that: a) IS needs a social environment to materialize, just like ES; b) Some types of ES can generate IS; c) The emergence of ES and IS does not depend on specific sectors; d) ES and IS bring changes in social practices; and e) Types of ES and IS can live together in a network environment. It was concluded that with social innovation being also caused by entrepreneurship, some implications can be observed when it comes to social management, especially regarding social participation in more systemic changes. It is recommended that in the studies of ES and IS, the paradigms being grounded are manifested in their texts, as well as philosophical positions or claims of knowledge so that it is possible to delineate a clearer epistemological path.

Keywords: Social Entrepreneurship; Social Innovation; Theoretical Approaches.
\end{abstract}

\section{Introdução}

Entende-se que o surgimento de estudos, aplicações de termos, de práticas e ações envolvendo Empreendedorismo Social (ES) e Inovação Social (IS), emergem de uma demanda crescente por respostas aos problemas sociais que de tempos em tempos vem sendo cada vez mais discutidos em razão de crises. Nas duas primeiras décadas dos anos 2000 muitos acontecimentos têm despertado questionamentos sobre a forma como a sociedade vem administrando suas tempestividades. Nesse instante, é preciso que se tenha uma ideia mais precisa do que realmente ES e IS podem contribuir nas mudanças sociais.

Todavia, acredita-se que até o momento muitos pesquisadores não tenham ainda definido muito bem os limites do entendimento do que é Empreendedorismo Social (ES) e Inovação Social (IS) ou até mesmo extrapolado estes limites. O que se encontra na literatura ainda é uma diversidade muito grande de indicações de trabalhos empíricos, mas com poucas definições e classificações.

O objetivo deste trabalho é discutir as conexões conceituais entre construtosna adoçãode umaperspectiva

Afiliação do autor:

Universidade Federal Rural de Pernambuco, Recife, Pernambuco, Brasil

*Email de correspondência: luizclaudio23@gmail.com 
empreendedora com foco em inovação social. Há um posicionamento comum entre pesquisadores de que quando se descreve o empreendedorismo social (ES) e a inovação social (IS) muitos consideram estes termos sinônimos (HULGÅRD; FERRARINI, 2010), entretanto neste artigo, eles serão tratados como aspectos distintos de uma ação maior para um bem comum.

Considerando que as inovações sociais emergem com o empreendedorismo, o que se questiona é: de que forma o ES faz surgir a IS? Todavia, há a possibilidade de uma mão-dupla nesta discussão em que a questão seria: de que forma a IS contribui para o ES? Alinhandose estas questões se tem um questionamento central que é: quais são as conexões conceituais entre ES e IS e a que se destinam,? Quais são as finalidades de ambos?

Então, a razão para a realização desta pesquisa é quanto ao problema da construção do conhecimento, vista a infinidade de aplicações que se utilizam para as práticas de ES e IS, que em certas circunstâncias podem estar equivocadas ou até mesmo sendo confundidas com outros termos já utilizados ou que simplesmente já foram discutidos e construídos em outras bases. Há, de certa forma, uma sensação de fragmentação dos estudos que impedem que haja uma consolidação das pesquisas.

Por fim, buscando reduzir a confusão teórica entre os termos, será feito aqui um esforço para a elucidação das convergências entre estas práticas. De maneira análoga, quando se trata da área de negócios convencionais, é visto que a origem do termo empreendedorismo é distinta da origem do termo inovação na visão econômica; entretanto, ambos têm aproximações que permitem a combinação de empreendedorismo e inovação de uma forma bastante sinérgica.

Para o alcance dos objetivos o trabalho traz, primeiro, a discussão do construto Empreendedorismo Social e, posteriormente, do construto Inovação Social, organizando as suas discussões conceituais e a descrição de finalidades, de modo que em ambos os termos possam ser verificados no que há de comum entre eles. Para sintetizar os achados, a partir da revisão de literatura, são construídos quadros que relacionam as pesquisas entre ES e IS.

\section{Empreendedorismo Social}

\section{Discussões iniciais}

A história do Empreendedorismo Social (ES) e as influências futuras aos seus estudos, tiveram forte ligação com o contexto norte-americano, europeu e asiático. No final da década de 1960, nos Estados Unidos da América (EUA), organizações sem fins lucrativos verificaram a necessidade de expandir suas atividades para preencher lacunas em seus orçamentos e, dessa forma, buscaram soluções a partir do uso de atividades comerciais num contexto onde havia uma grande desaceleração da economia e a oportunidade da participação destas organizações no Programa Grande Sociedade (RYAN, 1999).

$\mathrm{Na}$ Europa, as bases para o ES surgem do cooperativismo (KERLIN, 2006), que é um movimento já consolidado naquele continente, iniciado nos meados do século XIX, mas que começou a se fortalecer como uma prática de ES a partir da década de 1970 com a persistência do desemprego estrutural (PAQUÉ, 1998), a necessidade de redução de déficits orçamentários e as dificuldades de políticas sociais. Já na Ásia, a criação de novas estratégias organizacionais foi prioridade para Muhammad Yunus em 1976, dando início a formação de negócios sociais a partir do Grameen Bank (YUNUS, 2017), empreendendo em microfinanças por meio de empréstimos à população pobre de Bangladesh.

Esses contextos contribuíram para o crescimento do interesse pelo empreendedorismo social; entretanto, só na década de 1980 que o termo Empreendedor Social surge cunhado por Drayton da Ashoka, nos EUA (SEN, 2007), que considerava este tipo de empreendedor como o ator que poderia gerar inovação social. Na Europa surgiram oportunidades de empreender a partir do Programa de Políticas Ativas de Mercado (SCHÖMANN, 1995), encorajando a criação de novos empregos em razão do crescente desemprego e da crise nas finanças públicas. Neste período surge a New Ventures (DEFOURNY; NYSSENS, 2009) como consultoria para as NPOs (Non Profit Organizations) explorarem empreendimentos comerciais com o intuito de diversificar sua base de financiamento.

$\mathrm{Na}$ década seguinte, anos 1990, o termo empreendedorismo social ganha maior notoriedade e autores como Gregory Dees têm um papel relevante para a difusão do seu significado (DEFOURNY; NYSSENS, 2009). Na Ásia, a abertura de mercado na China contribuiu para a difusão de um ES que contemplava tanto características das iniciativas dos EUA quanto do Reino Unido (CURTIS, 2011). Além disso, foi na referida década que houve mundialmente um surgimento proeminente de organizações de fortalecimento do empreendedorismo social que tem sido emblemática nas ações de desenvolvimento destas iniciativas. Destacamse a EMES (Rede Europeia de Investigação) em 1996, a School for Social Entrepreneurs e a Young Foundation de Michael Young, no Reino Unido em 1997, a Schwab Foundation criada em 1998 na Suíça e no cenário norteamericano a criação da Skoll Foundation em 1999.

Nos anos 2000 houve uma grande profusão de artigos científicos (HILL; KOTHARI; SHEA, 2010), relatórios e outros tipos de publicações sobre ES e negócios sociais. Em 2009, no Reino Unido, a Global Entrepreneurship Research Association publicou um 
relatório especial da Global Entrepreneurship Monitor (GEM) dedicado ao Empreendedorismo Social, repetindo o mesmo feito em 2015 (TERJESEN et al, 2012; BOSMA et al. 2016).

\section{Discussões Emergentes}

Empreendedorismo social pode ser entendido como uma nova forma organizacional (BJÄRSHOLM, 2017), como práticas de soluções empresariais aplicadas a problemas sociais (GERMAK; ROBINSON, 2014), como um campo emergente e modelo de serviço transformador através de lentes teóricas de inovação (MUHAMAD; ADHAM, 2013).

Estas novas formas organizacionais têm um desafio gerencial muito grande, pois pretendem de certa forma conciliar perspectivas distintas de administração observadas em outros setores, como na Gestão Empresarial e na Gestão de Organizações do Terceiro Setor. Elas precisam combinar múltiplas lógicas institucionais, questões sociais não permitidas para empresas viáveis, dependência de demanda do consumidor e desvantagens competitivas quanto aos negócios convencionais (ROUNDY; BONNAL, 2017).

Também o ES pode ser compreendido como um processo de classificar problemas sociais para estabelecer um conjunto de soluções e um fator importante para inovação contínua (MULYANINGSIH; YUDOKO; RUDITO, 2016), como resultados da motivação e contexto dos empreendedores (NEWTH; WOODS, 2014) e também como uma inovação social (WITKAMP; RAVEN; ROYAKKERS, 2011).

Dessa maneira o empreendedorismo social, apesar de muitas vezes se iniciar de uma ideia individual do empreendedor, só atinge seus objetivos quando satisfaz as necessidades humanas por meio de ação coletiva. Isso envolve também considerar modelos distintos de construção e adequar cada estrutura a um tipo de necessidade, sem buscar um modelo único para a solução de todos os problemas sociais. Nos casos, por exemplo, de um regime de welfare state conservador, a estrutura mais adequada é o intraempreendedorismo (RUDDAT; SCHÖNAUER, 2014) em razão do caráter topdown das decisões deste tipo de regime.

Mas, ao considerar o ES como uma inovação social isto significa que ele é a própria mudança social. No entanto, as configurações de empreendedorismo social também passam por uma série de tipologias nas quais ele tende a ser mais facilmente discutido. Uma destas classificações são as empresas sociais (BARRAKET; FURNEAUX, 2012) que se são tidas como organizações que podem gerar inovação social.

Organizações da Sociedade Civil Chinesas vêm adotando abordagens empreendedoras e inovadoras para melhorar esta capacidade de cumprir a missão social (YU, 2016). Tais organizações não-lucrativas podem se transformar em socialmente empreendedoras participando de forma mais inovadora do que as convencionais, apesar da inovação de organizações não lucrativas socialmente empreendedoras não deixar de possuir desafios organizacionais e contextuais.

Além desta classificação, o empreendedorismo social pode assumir o formato de cooperativas, fundações e empresas híbridas, as quais podem operar separadas ou em conjunto num ambiente no modelo de parque empresarial (MURRAY; CAULIER-GRICE; MULGAN, 2010).

Outros ambientes também podem comportar o ES, principalmente ambientes de mercado. Grande parte deste interesse no ES tem sido visto em razão de suas beneficies e da sua sintonia com sinais mercadológicos. Já se percebe os empreendedores sociais como uma nova categoria de ator de mercado, apesar de certa dependência do intercâmbio voluntário, mas na maioria das vezes realizando a promoção da dignidade, a autossuficiência de beneficiários e a capacidade de explorar gastos do consumidor (ROUNDY; BONNAL, 2017).

Entretanto, frequentemente é visto que as universidades têm demonstrado um interesse muito grande em difundir práticas e teorias sobre o ES, seja por meio do desenvolvimento de habilidades, do envolvimento interpessoal, utilizando por vezes de uma abordagem única entre alunos, especialistas e professores (COOPER; GREENE, 2016) e em diferentes plataformas.

O Empreendedorismo Social (ES) como visto, pode ser descrito e entendido de várias formas. Mas, além disso, na literatura verifica-se que há também uma variedade de fins a que este termo tem sido vinculado. ES é associado a exploração de problemas sociais complexos, mas necessita de consistência entre elementos individuais, organizacionais e contextuais (PERRINI; VURRO; COSTANZO, 2010).

Dessa forma, a escolha do tipo de ES é algo que deve considerar como base, além de outros aspectos, como o impacto esperado de suas ações. Isto porque quanto mais inovador o ES mais impacto este pode gerar; ou seja, a estratégia de inovação serve ao ES para maior obtenção de valor social (WEERAWARDENA; MORT, 2012). Essa criação de valor pelo ES é importante para a economia (MÉNDEZ-PICAZO; RIBEIRO-SORIANO; GALINDO-MARTÍN, 2015).

Além disso, em razão das características dos empreendimentos sociais, estes podem tanto gerar mudança para os negócios quanto para o combate a vulnerabilidade de comunidades (ZEBRYTE; JORQUERA, 2017). De certo modo, sempre há uma variedade de organizações que gostariam do fim dos problemas sociais e que lutam por isso, uns contando com a contribuição de outros por meio de caridade e 
voluntariado para cumprir sua missão social e outros ofertando produtos e serviços dos quais têm seus ganhos revertidos para a missão social.

Percebe-se, então, que no ES há sempre visível um conflito entre a cultura da caridade e da solução de problemas sociais (DEES, 2012). Quando as organizações sociais por meio do ES conseguem cumprir sua missão, por vezes estes conseguem de certo modo promover o bem-estar (GROHS; SCHNEIDERS; HEINZE, 2015).

\section{Discussões Recentes}

O modo como empreendedores sociais desenvolvem soluções inovadoras para problemas sociais está associado a fatores como: antecipação, reflexividade, inclusão e deliberação das partes interessadas, capacidade de resposta e gestão do conhecimento (LUBBERINK et al 2018). Contudo, junto a estes aspectos pode ser inserida a dimensão de persistência nas dimensões genéricas da orientação empreendedora, pois apesar das dificuldades que empresas sociais passam principalmente em razão de riscos financeiros substanciais, elas inovam ao servir o propósito social e na maneira de obter renda porque tem o cuidado em não comprometer a missão social (SYRJÄ et al 2019).

Lidar com as tensões entre o paradoxo de criar valor social e econômico pode favorecer a adoção de diferentes estruturas organizacionais privilegiando uma dimensão ou outra na tomada de decisão. As empresas sociais podem gerar qualidade de vida, reduzir exclusão e fortalecer a economia (VAZQUEZ-MAGUIRRE; PORTALES, 2018).

Empreendedores sociais se concentram na criação de valor sócio ético direto para seus beneficiáriosalvo. Eles coordenam ações de stakeholders para desenvolver, implementar e dimensionar suas soluções. Suas inovações bottom-up são dimensionadas para obter impacto e buscam apoio institucional para criar mudanças top-down (LUBBERINK et al 2019).

\section{Inovação Social}

\section{Discussões Iniciais}

O termo inovação social foi mencionado primordialmente no início da década de 1970, nos Estados Unidos, trazendo aspectos fundamentais para a temática como a importância da comunidade, da participação da equipe e da responsabilidade igualitária (TAYLOR, 1970). Já na França, Chambon, David e Devevey (1982), publicam na Universidade da França, o livro: Les Innovations Sociales. Em 1986, Lévesque e Bélanger fundam no Canadá o CRISES (Centre de Recherche sur les Innovations Sociales) com o propósito de produção e disseminação de conhecimento, desenvolvimento de parcerias e treinamento de pesquisadores (CRISES, 2017).

Ainda nos anos 1980, nos EUA, Drucker (1987) tem seu artigo publicado sobre o tema, citando exemplos de IS dentro de um contexto de mudanças tecnológicas e comerciais para a época, com destaque para os Laboratórios de Pesquisa e os Movimentos de Massa, entre outros. Na década de 1990, o Centro de Inovação Social (ZSI) da Áustria entra em operação e em 1997 no Canadá é publicado o relatório do Mapa da Inovação contemplando a Inovação Social em um dos seus artigos.

Os anos 2000 foram de proeminência do tema, como visto a partir do lançamento da Stanford Social Innovation Review em 2003 pelo Nonprofit Management Institute (NMI) da Universidade de Stanford. Em 2006, em Singapura, Penny Low funda o Social Innovation Park (SIP, 2018) com soluções intersetoriais envolvendo o poder público, a iniciativa privada e a sociedade civil com o propósito de educar e capacitar empreendedores e inovadores sociais e, no ano seguinte também em Singapura, o Professor Arnoud De Meyer cria o centro de pesquisa Lien Centre for Social Innovation (LCSI, 2018).

Historicamente, percebe-se pelos fatos apresentados que a IS se concentrou em um primeiro momento em dois contextos distintos, o americano e o europeu. Nos EUA, por exemplo, a estagnação econômica era um fato que exigia respostas e mudanças sociais e econômicas. Do outro lado do mundo, na França, por exemplo, ocorria uma crise econômica que também era uma preocupação do Estado, mas seu governo socialista já havia programado o aumento de benefícios sociais. Contudo, a partir da década de 1990, as crises econômicas não foram os únicos motivos que geraram demandas por inovação social. Os avanços na ciência e tecnologia, as novas políticas econômicas e o efeito da globalização trouxeram novas possibilidades quanto a atuação das organizações na sociedade.

A partir do contexto em que foram construídas as suas ideias, compreende-se que o conceito de inovação social deve alinhar-se com o desenvolvimento das discussões intelectuais em curso sobre a sociedade (OSBURG; SCHMIDPETER, 2013) que envolvem mudanças ocorridas no pensamento econômico, político e acadêmico e a emergência de novas abordagens, conhecimentos e de esforços para a busca da sustentabilidade.

\section{Discussões Emergentes}

Martinelli et al. (2003) atribui a inovação social 
a um processo coletivo para necessidades humanas, mas também ela pode ser vista como um processo de aprendizado coletivo interativo bottom-up (RAONICHOLSON; VORLEY; KHAN, 2017) e além disso, tratar-se de um termo socialmente construído a partir de um contexto institucional (TURKER; ALTUNTASVURAL, 2017).

Mas, considera-se também a IS como um campo de ambiguidade conceitual, com uma diversidade de definições e configurações de pesquisa (VAN DER HAVE; RUBALCABA, 2016), além de ser um campo de pesquisa relativamente novo (PĂUNESCU, 2014). Dessa forma, a inovação social, assim como o empreendedorismo social compartilham de uma variedade de compreensões.

A inovação social possui esse caráter de mudança e quebra de paradigmas, de modo que ela se configura como um desafio ao mainstream dominante (SHAW; DE BRUIN, 2013), visto que propõe um foco maior no impacto social do que no econômico. Além disso, a IS pode ser entendida como um produto das expectativas financeiras, sociais, culturais e políticas de partes interessadas do empreendedorismo social (NEWTH; WOODS, 2014), assim como em muitos casos elas se tornam a base para empresas sociais cumprirem sua missão (MULYANINGSIH; YUDOKO; RUDITO, 2016) e atender expectativas.

Ao se falar sobre expectativas, sugere-se que a IS seja imaterial (CAJAIBA-SANTANA, 2014), pois seus resultados se manifestam por meio de mudanças de atitudes, comportamentos e práticas sociais. Assim, em certos casos, há dificuldades de mensuração em razão desta natureza e das várias dimensões que ela implica na sociedade.

$\mathrm{Na}$ sociedade, as inovações sociais (FRANZ; HOCHGERNER; HOWALDT, 2012) podem se concentrar tanto no estrato social, como no societal e até mesmo de forma sistêmica. Estas ocorrem a nível social quando há uma demanda urgente de natureza básica, ocorrem a nível societal quando o aspecto econômico é mais presente somado a um impacto mais amplo e ocorrem a nível sistêmico a partir de mudanças mais profundas, transformações em políticas, valores e/ou estruturas.

Nesse sentido, a IS permeia as camadas de ordem social, institucional e econômica, permeiam a estrutura social e modificam as características socioculturais. Estas mudanças podem ser promovidas por investimento corporativo para mudança, investimento em pessoal e liderança (SMUTCHAK; SYTNYK; OSTAPENKO, 2015).

Além destas camadas, a IS pode se desenvolver em projetos filantrópicos (MACLEAN; HARVEY; GORDON, 2013), trazendo abordagens inovadoras na forma de atuação de organizações que porventura necessitem de uma ação diferenciada para a busca de soluções de problemas sociais, que anteriormente não conseguiam ser resolvidos por abordagens mais convencionais.

Isto porque dentre suas finalidades principais estão aquelas que desencadeiam a melhoria do bem-estar (DAWSON; DANIEL, 2010), aquelas que contribuem para as cidades (SENENT-BAILACH; REY-MARTÍ, 2017) em diversos aspectos que podem incluir a inclusão social, como o exemplo no Impact Sourcing na forma de terceirização de indivíduos marginalizados, aos quais em muitas ocasiões não tem oportunidades de emprego (SANDEEP; RAVISHANKAR, 2015). Por outro lado, o que se percebe é que a IS pode ser integrada a determinadas estratégias de crescimento (SABATO; VANHERCKE; VERSCHRAEGEN, 2017) e trazer benefícios diretos às organizações que as constroem.

Por fim, as inovações sociais são construídas em diversas dimensões e em unidades distintas. Estas podem vir principalmente a partir de comunidades (TOIVONEN, 2016), podem ser promovidas por atores estabelecidos (GROHS; SCHNEIDERS; HEINZE, 2015), por empresas (SENENT-BAILACH; REYMARTÍ, 2017) e também pode ser promovida por meio do conhecimento produzido por empreendimento social solidário (GODÓI-DE-SOUSA; VALADÃO JÚNIOR, 2013).

Dessa forma, verifica-se também que com tantos atores, agentes e demais estruturas é percebida uma abordagem de sistemas de inovação que envolve tanto ES quanto IS (PHILLIPS et al, 2015) tornando-as parte de um objetivo maior.

Verificando tanto as finalidades de ES como de IS, percebe-se que em muitos pontos há convergências entre tais práticas. Além disso, alguns estudos mostram que esta junção é sinérgica e possui resultados mais impactantes. Nesse sentido, pode-se observar que, por exemplo, Comunidades de Inovação Social (CIS) são tidas como um apoio significativo para empreendimentos sociais em estágios iniciais (TOIVONEN, 2016). Nessa configuração, a IS contribui para o ES.

Em outro sentido, o ES pode promover IS quando empreendedores sociais estão focados na produção de conhecimento, que, de certa forma, teria como finalidade o desenvolvimento intelectual (GODÓI-DE-SOUSA; VALADÃO JÚNIOR, 2013). Quando a IS também é integrada como estratégia de crescimento de um país, estado ou território, o ES tem sido visto como uma fase desse processo dentro da referida estratégia (SABATO; VANHERCKE; VERSCHRAEGEN, 2017).

Ao analisar as contribuições aos estudos de ES, percebe-se ainda que existem impedimentos ao seu avanço teórico; estes impedimentos estão relacionados aos limites que muitos estudos impõem para o tema e que deveriam ser avaliados onde a teoria deve ser agregada ou desagregada, já que existem tópicos que promovem a pesquisa (MUELLER et al., 2015) e outros 
que podem confundir a trilha epistemológica que deve ser percorrida.

Dessa maneira, esses limites precisam ser avaliados quando se percebe que há convergências, como visto, entre ES e IS, como a implícita orientação normativa do ES e dos processos de IS (MUELLER et al., 2015).

\section{Discussões Recentes}

As inovações podem ser classificadas e identificadas a partir de suas características, assim podem existir do tipo produto, organizacional ou marketing, com profundidade, incremental, disruptiva e institucional (DAMARIO; COMINI, 2020), realizadas em diferentes âmbitos geográficos. Essas inovações sociais podem ser difundidas e mobilizadas por organizações sociais por meio de serviços aos quais contribuem diretamente com desenvolvimento das próprias organizações sociais (MUHAMAD et al., 2018).

Seja em organizações sociais ou em outros modos de gerar inovação social as iniciativas precisam de um projeto. A partir disso, acredita-se que a eficiência econômica da implementação de um projeto social depende de benefícios comerciais e públicos e devem estar descritos em uma matriz de avaliação do impacto da inovação social. Ao avaliar o impacto das inovações sociais é possível promover uma modelagem institucional abrangente de desenvolvimento tanto do setor público quanto de outras forças motrizes (POPOV, 2018).

As forças motrizes para a inovação social podem vir de um sistema de inovação. Entretanto, é importante ressaltar que um sistema de inovação deve se concentrar na solução de problemas de um modo mais abrangente, em que seja preciso uma combinação de inovações técnicas que ataquem os aspectos tecnológicos do problema e inovações sociais que deem enfoque aos aspectos sociais do mesmo problema (GHAZINOORY et al., 2020).

\section{Procedimentos Metodológicos}

Os procedimentos metodológicos seguiram para uma revisão sistemática qualitativa com enfoque meta-analítico (MARIANO; CRUZ; GAITÁN, 2011) utilizando a análise de conteúdo para extrair temas dos textos que tratam tanto de inovação social como empreendedorismo social. Nesse sentido, o trabalho traz, a partir dos seus resultados, a análise de dados obtidos por temas frequentes, mas também relevantes no intuito de trazer exemplos da literatura de formas de empreendedorismo social que geraram inovação social.

A partir dos anos 2000, milhares de artigos científicos trataram de ES ou IS trazendo um universo de trabalhos que tornaria difícil uma análise mais dinâmica da história entre as convergências destes construtos; contudo, quando se busca a junção das discussões destes termos ao mesmo tempo, em um trabalho científico, esse universo é reduzido e a eficiência da busca por pontos em comum se torna mais focada. Dessa forma, foi feita uma pesquisa com os termos "social entrepreneurship" e "social innovation", acrescentando-se o conector "and" e limitando sua frequência principal nos títulos e palavras-chave.

Além disso, para consolidar mais rapidamente todas as coletas, foi escolhida uma das bases de dados mais indicadas para revisões sistemáticas, neste caso a Scopus, onde a exportação dos dados em conjunto foi feita para proceder a revisão. A maioria dos artigos foi escrita em língua inglesa, com exceção de 2 artigos em russo, 2 em francês e 5 em língua espanhola.

Empreendedorismo Social (ES) e Inovação Social (IS) são construtos que possuem conexões conceituais, as quais podem ser identificadas a partir da revisão de literatura em seus casos teóricos empíricos ao longo do tempo. Pressupõe-se que do lado social as aproximações estejam ligadas ao objetivo principal de cada prática e do lado do empreendedorismo e inovação suas ligações estejam relacionadas a forma diferenciada das ações em relação aos padrões estabelecidos e às tradições. As relações entre os construtos podem partir de ambos os sentidos, seguindo tanto do ES para IS quanto o contrário; vide Quadro 1.

Os dados secundários obtidos da literatura de ES e IS foram tratados agregando 100 artigos extraídos na base de dados Scopus a partir da seleção de campos como: autor (es), título, ano de publicação, fonte, número de citações, instituição afiliada, palavras-chave e linguagem original do texto. A partir disso, os artigos foram classificados segundo sua aderência para união entre os construtos ES e IS, sendo segmentados em 4 categorias de artigos: aderência total, aderência alta, aderência mediana e baixa aderência.

Num primeiro momento foram selecionados apenas os artigos de total e alta aderência somando 47 artigos; entretanto, foi realizada uma segunda avaliação que extraiu dos artigos de aderência mediana mais 4 trabalhos que estavam mais próximos da alta aderência. Assim, ao final ficaram 51\% dos trabalhos selecionados na base de dados.

O fim do processo metodológico contou com a Análise de conteúdo (BARDIN, 2011) que foi realizada de maneira categorial, de modo que puderam ser construídos 2 quadros resumindo contribuições para as aproximações entre os construtos e as finalidades observadas entre ES e IS. Em razão da natureza dos dados em cada etapa, para o Quadro 2 de aproximações foi realizado 1 nível de codificação e para o Quadro 3 de finalidades foram realizados 2 níveis de codificação. 
Quadro 1. Relação bilateral de ES e IS

\begin{tabular}{|c|c|c|}
\hline Construto & Empreendedorismo Social (ES) & Social \\
\hline Origens & Empreendedorismo $x$ Inovaçẵo & Impal (IS) \\
\hline Finalidade & Quebra de paradigma & Impacto nociedade \\
\hline
\end{tabular}

\section{Resultados e Análises}

A partir da revisão sistemática qualitativa da literatura entre ES e IS foi possível construir 2 quadros resumindo contribuições para as aproximações entre os construtos e as finalidades observadas entre eles. No Quadro 2 pode ser vista aproximação entre o entendimento sobre ES e IS e, por vezes, o resultado dessa aproximação em conjunto.

Aproximações entre ES e IS ocorrem porque ambos são considerados campos de pesquisa (C01) e, portanto, em um mesmo patamar de relevância para discutir soluções sociais. Dessa forma, muitos dos seus produtos ou resultados (C02) são esperados por determinado grupo social independente se são ações de uma ou de outra prática. No entanto, tal tratamento também traz impressões de que possam ser a mesma coisa.

ES e IS tem na sua base de discussão as soluções de problemas sociais $(\mathrm{C} 03)$ e para isso se utilizam mais de processos (C04) do que produtos. Dessa forma, o gerenciamento do processo, a forma como tudo é feito em grande parte é tão importante quanto o resultado. Diferente, muitas vezes, em relação aos negócios convencionais em que muitos consumidores não se importam de como é feito tal produto ou os processos que estão na retaguarda dos serviços.

ES e IS, em razão das suas práticas de missão social e a valorização da participação de todos os interessados em ambas as atividades, tendem a ser termos socialmente construídos dentro de um contexto institucional (05); ou seja, além de um processo coletivo envolvendo o fortalecimento da ação social, existe uma legitimação que lhe é dada no contexto em que se insere.

A configuração tanto de ES e IS e sua relevância tanto para sociedade quanto para a economia permitem que possam ser considerados como componentes de um sistema de inovação (C06), seja local, regional ou nacional, dependendo do seu alcance, das áreas e tipo de produto ou serviço que está sendo ofertado. De maneira geral essa configuração sustentável é chamada de ecossistema.

Ambos, ES e IS tem em seu escopo uma busca equilibrada para a integração sistemática entre sociedade civil e mercados (C07) de maneira que harmonicamente estes entes possam contribuir para o atingimento da missão, trazendo o melhor que cada processo pode obter para o alcance da eficiência, eficácia e efetividade das práticas sociais.

O mainstream dominante de empreendedorismo (C08) é tido como as práticas de empreendedorismo convencionais que partem dos pilares das ações de agentes econômicos e que tem como principal objetivo o ganho econômico. Entretanto, organizações híbridas que se utilizam tanto de ES como de IS são um desafio para romper a lógica dominante.

Apesar de já ter sido mencionado que os termos ES e IS são em grande parte fruto de uma construção social, as características de processo em que se inserem, muitas vezes indicam certa orientação normativa implícita (C09), principalmente em casos em que há uma necessidade posterior de difusão das práticas de empreendedorismo e inovação sociais.

A presença de ES e IS na educação traz consigo a indicação de que as práticas que são socialmente construídas devem fazer parte da transformação da sociedade em seus aspectos mais relevantes e, dessa forma, sua introdução nas universidades propõe também um novo currículo para a formação profissional, seja nas abordagens práticas presenciais ou online (C10).

Apesar do discurso às vezes heroico que envolve empreendedores e inovadores sociais, não há uma eliminação definitiva de desafios organizacionais, mas há sim uma redução (C11), a qual se percebe através da união de perspectivas distintas em prol da conciliação destas para melhor atender a sociedade.

Por fim, o que foi visto que em relação a modelos anteriores de organização para fins sociais, foi uma melhoria da capacidade, seja de Organizações da Sociedade Civil ou Organizações não lucrativas etc. (C12) para atender os seus propósitos, trazendo para estas um rol de ferramentas que auxiliam e contribuem para um melhor gerenciamento das organizações de maneira que possam ter opções para decisão e implementação de novas ideias.

Já o Quadro 3 sintetiza finalidades de ES e IS, mas também mostrando o foco de cada finalidade apresentada dentro de 4 grandes dimensões: Comunidade, Economia, Sociedade e Organizações. Estas dimensões representam o foco que cada fim de ES e IS puderam ser agrupados. 
Quadro 2. Tipos de aproximações conceituais encontradas na literatura

\begin{tabular}{|c|c|c|c|}
\hline $\begin{array}{c}\text { Empreendedorismo Secial } \\
\text { (RS) }\end{array}$ & Inovaçăo Social (IS) & Tipo de Aproximaçalo & Cód. \\
\hline $\begin{array}{l}\text { Considerado como campo de } \\
\text { pesquisa }\end{array}$ & $\begin{array}{l}\text { Considerado como eampo de } \\
\text { pesquisa }\end{array}$ & $\begin{array}{l}\text { Ambos congregam pesquisas } \\
\text { como num campo de } \\
\text { conhecimento }\end{array}$ & $\mathrm{COl}$ \\
\hline $\begin{array}{l}\text { Resultados de motivaçâto de } \\
\text { empreendedores }\end{array}$ & $\begin{array}{l}\text { Produto de expectativa dos } \\
\text { stakebolders }\end{array}$ & $\begin{array}{l}\text { Produtos ou resultados } \\
\text { esperados por determinado } \\
\text { grupo social }\end{array}$ & $\mathrm{CO} 2$ \\
\hline $\begin{array}{l}\text { Soluçōes empresarias para } \\
\text { problemas sociais }\end{array}$ & $\begin{array}{l}\text { Base para empresas sociais } \\
\text { cumprirem sua missìo }\end{array}$ & $\begin{array}{l}\text { Base para soluçōes de problemas } \\
\text { sociais }\end{array}$ & $\mathrm{CO}$ \\
\hline $\begin{array}{l}\text { Processo de classificação de } \\
\text { problemas sociais para busca de } \\
\text { soluções }\end{array}$ & $\begin{array}{l}\text { Processo de aprendizado } \\
\text { coletivo interativo de baixo } \\
\text { para cima (bottom up); }\end{array}$ & $\begin{array}{l}\text { Processos que visam soluçōes e } \\
\text { que se alicerçam em saberes }\end{array}$ & $\mathrm{CO4}$ \\
\hline $\begin{array}{l}\text { Modelo de serviço } \\
\text { transformador-inovador }\end{array}$ & $\begin{array}{l}\text { Termo socialmente } \\
\text { construido dentro de um (CT) } \\
\text { contexto institucional; }\end{array}$ & $\begin{array}{l}\text { Termos socialmente construidos } \\
\text { dentro de um } \mathrm{CI}\end{array}$ & $\cos$ \\
\hline Novo modelo de negócios & $\begin{array}{l}\text { Tipo de ação empresarial } \\
\text { inovadora }\end{array}$ & $\begin{array}{l}\text { Componentes de um sistema de } \\
\text { inovaçào }\end{array}$ & C06 \\
\hline $\begin{array}{l}\text { Nova categoria de atores de } \\
\text { mercado }\end{array}$ & $\begin{array}{l}\text { Fatoe de sucesso de } \\
\text { determinadas empresas }\end{array}$ & $\begin{array}{l}\text { Integração sistemática entre } \\
\text { sociedade civil e mercados }\end{array}$ & $\mathrm{CO} 7$ \\
\hline $\begin{array}{l}\text { Fase da IS, Processo de IS, Tipo } \\
\text { de IS, Inovaçũo Social }\end{array}$ & Prática de ES & $\begin{array}{l}\text { ES e IS como desafio ao } \\
\text { mainstream dominante de } \\
\text { empreendedorismo }\end{array}$ & $\mathrm{Cos}$ \\
\hline $\begin{array}{l}\text { Um modelo não tăo fácil de } \\
\text { transferir }\end{array}$ & $\begin{array}{l}\text { Pode surgir de regime de } \\
\text { bem-estar conservador sem } \\
\text { ES }\end{array}$ & $\begin{array}{l}\text { Orientaçäo normativa implicita } \\
\text { em suas iniciativas }\end{array}$ & $\mathrm{C} 09$ \\
\hline $\begin{array}{l}\text { Interesse de educaçăo nas } \\
\text { Universidades }\end{array}$ & $\begin{array}{l}\text { Investimento corporativo em } \\
\text { pessoas }\end{array}$ & $\begin{array}{l}\text { Ligação forte com valorizaçăo } \\
\text { do capital intelectual }\end{array}$ & $\mathrm{ClO}$ \\
\hline $\begin{array}{l}\text { Forma organizacional; } \\
\text { Combinação de múltiplas } \\
\text { Lógicas institucionais }\end{array}$ & $\begin{array}{l}\text { Mobilizadas por } \\
\text { Organizą̧es Sociais }\end{array}$ & $\begin{array}{l}\text { Focum em reduçĩo de dilemas } \\
\text { organizacionais }\end{array}$ & C11 \\
\hline $\begin{array}{l}\text { Negócio com desvantagem } \\
\text { compctitiva cm relaçăo aos } \\
\text { negócios convencionais }\end{array}$ & $\begin{array}{l}\text { Não possui enfoxque em } \\
\text { vantagem competitiva }\end{array}$ & $\begin{array}{l}\text { Melhoria da capacidade de } \\
\text { organizações com missão social }\end{array}$ & $\mathrm{Cl} 2$ \\
\hline
\end{tabular}

A partir do Quadro 2 se verificou que há uma considerável quantidade de pontos de convergência nas conexões conceituais entre ES e IS e entre suas finalidades (Quadro 3) no que concerne o aspecto geral em que estas estão incluídas. Estes pontos puderam ser categorizados em 4 grupos: comunidade, economia, sociedade e organizações.

Quanto à comunidade, a finalidade do ES é a 
mudança da realidade destes coletivos e a consequência destes atos podem ser as mais diversas possíveis; entretanto, se houver uma coesão significativa tanto de conhecimento, práticas e comportamentos dos indivíduos que compõe a comunidade, e até mesmo de colabores externos, temos que esta poderá se tornar uma Comunidade de Inovação Social (CIS).

Já em relação à economia, em termos de finalidade, ambos têm implicações neste aspecto, mas com uma atuação mais econômica do ES e uma ação mais social e a valorização de aspectos não econômicos pelo IS. Nesse sentido, os destaques vão para: promoção da dignidade e autossuficiência dos beneficiários a partir de ações empreendedoras e a contribuição para as cidades quando se geram inovações sociais.

Quanto à sociedade, o ES contribui para a exploração de problemas sociais complexos, para a promoção de bem-estar e para a criação de valor social, assim como a IS promove a melhoria do bem-estar e foca na abordagem de problemas sociais, principalmente aqueles mais enraizados. Em geral, a IS traz a criação de impacto significativo por um ecossistema interativo e busca de várias formas explicar mudanças sociais. Além disso, a IS pode ser promovida pelo ES por meio da produção de conhecimento.

Em relação às organizações, quando se trata de ES este surge como uma alternativa à cultura de caridade para a obtenção de maior impacto social, buscando ser mais viável, mas mantendo o foco no social e na mudança nos negócios. De uma forma similar a IS surge como uma alternativa de estratégia para projetos filantrópicos ou para integrar a estratégia de crescimento de outros tipos de projetos, trazendo possibilidades para a obtenção de impacto social significativamente maior e focando em inovações inclusivas como o Impact Sourcing.

\section{Considerações Finais}

Empreendedorismo Social e Inovação Social ambos são considerados campos de pesquisa em razão da relevância que tem seus estudos, razão por que é

Quadro 3. Rol de Finalidades e Contribuições advindas da revisão sistemática.

\begin{tabular}{|c|c|c|}
\hline Foco & Empreendedorismo Social (ES) & Inovaçâo Social (IS) \\
\hline $\begin{array}{l}\text { Quanto à } \\
\text { comunidade }\end{array}$ & Provocar mudança cm comunidades vulneráveis & $\begin{array}{l}\text { Organizar Comunidades de Inovaçĩo } \\
\text { Sociul (CIS) }\end{array}$ \\
\hline \multirow{2}{*}{$\begin{array}{l}\text { Quanto í } \\
\text { cconomia }\end{array}$} & Contribuir na melhoria do elima econômico & $\begin{array}{l}\text { Focar na mudança social e no } \\
\text { reconhecimento dos valores nậo } \\
\text { económicos }\end{array}$ \\
\hline & $\begin{array}{l}\text { Promover dignidade c autossuficiência dos } \\
\text { beneficiários }\end{array}$ & $\begin{array}{l}\text { Atentar para a contribuiçăo para as } \\
\text { cidades }\end{array}$ \\
\hline \multirow{4}{*}{$\begin{array}{l}\text { Quanto à } \\
\text { sociedade }\end{array}$} & $\begin{array}{l}\text { Explorar resoluçio de problemas sociais } \\
\text { complexos }\end{array}$ & Abordar problemas sociais enraizados \\
\hline & Promover bem-estar & Proporcionar melhoria do bem-estar \\
\hline & $\begin{array}{l}\text { Promover IS por meio da produção de } \\
\text { conhecimento }\end{array}$ & Explicar mudanças sociais \\
\hline & Criar valor social & $\begin{array}{l}\text { Criar impacto significativo por meio de } \\
\text { um ecossistema interativo }\end{array}$ \\
\hline \multirow{4}{*}{$\begin{array}{l}\text { Quanto às } \\
\text { organizaçōes }\end{array}$} & $\begin{array}{l}\text { Elaborar alternativas sustentáveis à cultura de } \\
\text { caridade }\end{array}$ & $\begin{array}{l}\text { Inovar na estratégia para projetos } \\
\text { filantrópicos }\end{array}$ \\
\hline & $\begin{array}{l}\text { Gerenciar uma maior obtenção de impacto } \\
\text { social }\end{array}$ & $\begin{array}{l}\text { Obter impacto social significativamente } \\
\text { maior que outrus iniciativas }\end{array}$ \\
\hline & Sustentar o caráter viável com foco no social & Dirigir ap̧bes de Impact Sourcing \\
\hline & Mudar a forma de fazer negócio & Integrar-se às estratégias de negócios \\
\hline
\end{tabular}


preciso verificar em quais bases tais campos podem ser inseridos para que tenham uma mesma linha de trabalho ou agenda. Por serem resultados esperados por determinados grupos sociais é relevante que se insiram estudos sobre expectativas de beneficiários ou clientes de maneira que a gestão do relacionamento com o público seja um fator crítico de sucesso.

Práticas de ES e IS nos tempos modernos se tornaram, em muitos casos, a base para soluções de problemas sociais e como sua abordagem é voltada para processos na maioria das vezes isto exige que tenham uma orientação normativa implícita (C09). A construção social que se percebe quando se fala destes termos dentro de cada contexto institucional traz a sensação de que há uma forte necessidade de uma base sociológica que explique suas práticas.

Em razão da integração sistemática entre sociedade civil e mercados promovidas por práticas socialmente empreendedoras estas tornam-se importantes componentes de um sistema de inovação. Este sistema necessita de uma presença forte das instituições de ensino, principalmente as de ensino superior, que promovem também pesquisa e extensão, já que ES e IS tem sido visto muito presentes na educação (C10).

ES e IS são um desafio às tendências de ações empreendedoras voltadas para fins exclusivamente econômicos, visto que nem sempre são tidos como alternativas viáveis economicamente (C08). Por outro lado, como a economia possui três setores, quando se beneficia um destes há grandes chances de se ter impactos nos outros. Assim sendo, verificam-se em casos empíricos os benefícios de melhoria da capacidade de OSC e uma redução em alguns desafios organizacionais (C11).

Dentre os aspectos que encontramos como maiores aproximações entre ES e IS são, em suma, que: a) a IS precisa de um meio social para se concretizar, assim como a ES (C05), pois são construídos socialmente e institucionalmente; b) alguns tipos de ES podem gerar IS (C12) em razão da melhoria que trouxeram para a capacidade OSC em muitos casos; c) o surgimento de ES e IS independem de setores específicos (C07) já que se tratam de uma integração entre sociedade civil e mercados; d) ES e IS trazem mudanças nas práticas sociais (C03), pois perseguem soluções de problemas sociais; e) tipos de ES e IS podem conviver conjuntamente em um ambiente de rede (C06) por serem consideradas componentes de um sistema de inovação.

Ao serem retratadas as finalidades de ES e IS e a aderência entre elas, algumas descobertas podem ser vistas:

1) As práticas de ES e IS em razão do seu foco social levam a crer que suas contribuições são apenas sociais, mas o que se percebe é que elas se expandem a outros níveis além da comunidade. Vê-se, então, contribuições diretas para a economia e sociedade tanto local quanto global dependendo do tipo de alcance que tenha tido;

2) As formas como ES e IS buscam maior eficiência e sustentabilidade de suas ações, além de outros aspectos característicos destas práticas, trazem às organizações contribuições que podem ser aproveitadas por outros tipos de empreendimentos e projetos com foco mais lucrativo.

Foi verificado que as conexões conceituais de ambos os construtos têm sentidos bilaterais e estas associações partem tanto do ES para o IS como também do IS para o ES. Concluiu-se que com a inovação social sendo também causada pelo empreendedorismo algumas implicações podem ser observadas quando se trata da gestão social (TENÓRIO, 2008), principalmente no que concerne a participação social nas mudanças mais sistêmicas.

A partir da revisão sistemática qualitativa da literatura entre ES e IS foi possível construir 2 quadros resumindo contribuições para as aproximações entre os construtos e as finalidades observadas entre eles. No Quadro 2 pode ser vista aproximação entre o entendimento sobre ES e IS e, por vezes, o resultado dessa aproximação em conjunto.

\section{Referências}

BARDIN, L. Análise de conteúdo. 3. reimp. Lisboa: Ediçoes 70, 2011.

BARRAKET, J.; FURNEAUX, C. Social innovation and social enterprise: evidence from Australia. Challenge Social Innovation. Springer Berlin Heidelberg, p. 215-237, 2012.

BJÄRSHOLM, D. Sport and social entrepreneurship: A review of a concept in progress. Journal of Sport Management, v. 31, n. 2, p. 191-206, 2017

BOSMA, N. et al. Global Entrepreneurship Monitor 2015 to 2016: Special Topic Report Social Entrepreneurship. Global Entrepreneurship Research Association, 2016.

CAJAIBA-SANTANA, G. Social innovation: Moving the field forward. A conceptual framework. Technological Forecasting and Social Change, v. 82, p. $42-51,2014$

CHAMBON, Jean-Louis; DAVID, Alix; DEVEVEY, Jean-Marie. Les innovations sociales. Presses universitaires de France, 1982.

COOPER, C.; GREENE, J. Making Social Innovation and Social Entrepreneurship Education More Accessible and Relevant. The International Journal of Science, Mathematics and Technology Learning, Aug. 2016.

CRISES, Centre de recherche sur les innovations sociales. Présentation. Disponível em: <http://crises.uqam.ca/le-centre/presentation.html>. Acesso em: 18 Set 2017.

CURTIS, Timothy. 'Newness' in social entrepreneurship discourses: the concept of 'danwei'in the Chinese experience. Journal of Social Entrepreneurship, v. 2, n. 2, p. 198-217, 2011.

DAMARIO, E. Q.; COMINI, G. M. Social Innovation in Brazilian Social Entrepreneurships: A Proposed Scale for its Classification. Revista Brasileira de Gestão de Negócios, v. 22, n. 1, p. 104-122, 2020.

DAWSON, P.; DANIEL, L. Understanding social innovation: a provisional framework. International Journal of Technology Management, v. 51, n. 1, p. 9-21, 2010. 
DEES, J. G. A tale of two cultures: Charity, problem solving, and the future of social entrepreneurship. Journal of business ethics, v. 111, n. 3, p. 321$334,2012$.

DEFOURNY, Jacques; NYSSENS, Marthe. Social enterprise: The shaping of a new concept in a comparative regional perspective. The Worldwide Making of the Social Economy. Innovations and Changes, p. 265-291, 2009.

DEFOURNY, Jacques; NYSSENS, Marthe. Social enterprise: The shaping of a new concept in a comparative regional perspective. The Worldwide Making of the Social Economy. Innovations and Changes, p. 265-291, 2009.

DRUCKER, Peter F. Social innovation-management's new dimension. Long Range Planning, v. 20, n. 6, p. 29-34, 1987.

FRANZ, H-W.; HOCHGERNER, J.; HOWALDT, J. (ed.). Challenge social innovation: potentials for business, social entrepreneurship, welfare, and civil society. Springer Science \& Business Media, 2012.

GERMAK, A. J.; ROBINSON, J. A. Exploring the motivation of nascent social entrepreneurs. Journal of Social Entrepreneurship, v. 5, n. 1, p. 5-21, 2014

GHAZINOORY, S. et al. Why do we need 'Problem-oriented Innovation System (PIS)'for solving macro-level societal problems? Technological Forecasting and Social Change, v. 150, p. 119749, 2020.

GODÓI-DE-SOUSA, E.; VALADÃO JÚNIOR, V. M. Social Enterprises in Brazil: Socially Produced Knowledge Versus Social Innovation. Journal of Technology Management \& Innovation, v. 8, p. 166-176, 2013.

GROHS, S.; SCHNEIDERS, K.; HEINZE, R. G. Social entrepreneurship versus intrapreneurship in the German social welfare state: A study of oldage care and youth welfare services. Nonprofit and voluntary sector quarterly, v. 44, n. 1, p. $163-180,2015$.

HILL, T. L.; KOTHARI, Tanvi H.; SHEA, Matthew. Patterns of meaning in the social entrepreneurship literature: A research platform. Journal of social entrepreneurship, v. 1, n. 1, p. 5-31, 2010.

HULGÅRD, L.; FERRARINI, A. V. Inovação social: rumo a uma mudança experimental na política pública? Ciências Sociais Unisinos, v. 46, n. 3, p. 256-263, 2010.

KERLIN, Janelle A. Social enterprise in the United States and Europe: Understanding and learning from the differences. Voluntas: International Journal of Voluntary and Nonprofit Organizations, v. 17, n. 3, p. 246, 2006.

KERLIN, Janelle A. Social enterprise in the United States and Europe: Understanding and learning from the differences. Voluntas: International Journal of Voluntary and Nonprofit Organizations, v. 17, n. 3, p. 246, 2006.

LCSI, Lien Centre for Social Innovation. Abou Us. Disponível em: $<$ https:// lcsi.smu.edu.sg/ >. Acesso em: 18 Ago 2018.

LUBBERINK, R. et al. Innovation for society: Towards a typology of developing innovations by social entrepreneurs. Journal of Social Entrepreneurship, v. 9, n. 1, p. 52-78, 2018.

LUBBERINK, R. et al. Responsible innovation by social entrepreneurs: an exploratory study of values integration in innovations. Journal of Responsible Innovation, v. 6, n. 2, p. 179-210, 2019.

MACLEAN, M.; HARVEY, C.; GORDON, J. Social innovation, social entrepreneurship, and the practice of contemporary entrepreneurial philanthropy. International Small Business Journal, v. 31, n. 7, p. 747-763, 2013.

MARIANO, A. M.; CRUZ, R. G.; GAITÁN, J. A. Meta análises como instrumento de pesquisa: Uma revisão sistemática da bibliografia aplicada ao estudo das alianças estratégicas internacionais. In: Congresso Internacional de Administração-Inovação Colaborativa e Competitividade. 2011.

MARTINELLI, F. et al. Social Innovation, Governance, and Community Building. SINGOCOM. Report April, 2003.

MÉNDEZ-PICAZO, M.-T; RIBEIRO-SORIANO, D.; GALINDOMARTÍN, M.-Á. Drivers of social entrepreneurship. European Journal of International Management, v. 9, n. 6, p. 766-779, 2015.

MUELLER, S. et al. What's Holding Back Social Entrepreneurship? Removing the Impediments to Theoretical Advancement. Journal of social entrepreneurship, v. 6, n. 3, p. 245-256, 2015.
MUHAMAD, N. S. et al. How Does Transformative Social Service Develop? Journal Pengurusan (UKM Journal of Management), v. 54, 2018.

MUHAMAD, N. S.; ADHAM, K. A. Social entrepreneurship as transformative service for societal well-being. Jurnal Pengurusan. (UKM Journal of Management), v. 39, 2013.

MULYANINGSIH, H. D.; YUDOKO, G.; RUDITO, B. Knowledge-Based Social Innovation Process in Social Enterprise: A Conceptual Framework. Advanced Science Letters, v. 22, n. 5-6, p. 1393-1397, 2016.

MURRAY, R.; CAULIER-GRICE, J.; MULGAN, G. The open book of social innovation. London: National Endowment for Science, Technology and the Art, 2010.

NEWTH, J.; WOODS, C. Resistance to social entrepreneurship: How context shapes innovation. Journal of Social Entrepreneurship, v. 5, n. 2, p. 192-213, 2014

OSBURG, Thomas; SCHMIDPETER, René. Social innovation. Solutions for a sustainable future. Springer, 2013.

PAQUÉ, Karl-Heinz. Structural Unemployment in Europe: A Bird's-Eye View. In: Labor markets and social security. Springer, Berlin, Heidelberg, 1998. p. $17-48$

PĂUNESCU, C. Current trends in social innovation research: social capital, corporate social responsibility, impact measurement. Management \& Marketing, v. 9, n. 2, p. 105, 2014.

PERRINI, F.; VURRO, C; COSTANZO, L. A. A process-based view of social entrepreneurship: From opportunity identification to scaling-up social change in the case of San Patrignano. Entrepreneurship and Regional Development, v. 22, n. 6, p. 515-534, 2010.

PHILLIPS, Wendy et al. Social innovation and social entrepreneurship: A systematic review. Group \& Organization Management, v. 40, n. 3, p. 428461,2015

POPOV, E. V. Econotronics. Regional Economy, v. 14, n. 1, p. 13-28, 2018.

RAO-NICHOLSON, R.; VORLEY, T.; KHAN, Z. Social innovation in emerging economies: A national systems of innovation-based approach. Technological Forecasting and Social Change, v. 121, p. 228-237, 2017.

ROUNDY, P. T.; BONNAL, M. The Singularity of Social Entrepreneurship: Untangling its Uniqueness and Market Function. The Journal of Entrepreneurship, v. 26, n. 2, p. 137-162, 2017.

RUDDAT, C.; SCHÖNAUER, A-L. New players on crowded playing fields: the institutional embeddedness of social innovation in Germany. Social Policy and Society, v. 13, n. 3, p. 445-456, 2014.

RYAN, William P. The new landscape for nonprofits. Havard Business Review, Jan-Feb, 1999.

SABATO, S.; VANHERCKE, B.; VERSCHRAEGEN, G. Connecting entrepreneurship with policy experimentation? The EU framework for social innovation. Innovation: The European Journal of Social Science Research, v. 30 , n. 2, p. 147-167, 2017.

SANDEEP, M. S.; RAVISHANKAR, M. N. Social innovations in outsourcing: An empirical investigation of impact sourcing companies in India. The Journal of Strategic Information Systems, v. 24, n. 4, p. 270-288, 2015.

SCHÖMANN, Klaus. Active labour market policy in the European Union. WZB Discussion Paper, 1995.

SEN, Pritha. Ashoka's big idea: Transforming the world through social entrepreneurship. Futures, v. 39, n. 5, p. 534-553, 2007.

SENENT-BAILACH, C.; REY-MARTÍ, A. Creating Entrepreneurship through Social Innovation: The Case of I-Box Create. Contemporary Economics, v. 11, n. 4, 2017.

SHAW, Eleanor; DE BRUIN, Anne. Reconsidering capitalism: the promise of social innovation and social entrepreneurship? International Small Business Journal, v. 31, n. 7, p. 737-746, 2013.

SIP, Social Innovation Park. About Us. Disponível em: <http://www. socialinnovationpark.org/>. Acesso em: 18 Ago 2018. 
SMUTCHAK, Z.; SYTNYK, O.; OSTAPENKO, O. Activation of social innovations as a premise for development of human resources. Economic Annals-XXI, 2015.

SYRJÄ, P. et al. Entrepreneurial orientation in firms with a social mission-a mixed-methods approach. Cogent Business \& Management, v. 6, n. 1, p. $1602016,2019$.

TAYLOR, James B. Introducing social innovation. The journal of applied behavioral science, v. 6, n. 1, p. 69-77, 1970.

TENÓRIO, F. G. A trajetória do Programa de Estudos em Gestão Social (PEGS). In: SILVA JR., J. T. (Org.) et al. Gestão Social: Práticas em debate, teorias em construção. Fortaleza: Imprensa Universitária, 2008.

TERJESEN, S. et al. Global entrepreneurship monitor: 2009 report on social entrepreneurship. Santiago: Babson Park, London: Babson College, Universidad del Desarrollo, Global Entrepreneurship Research Association, 2012.

TOIVONEN, T. What is the social innovation community? Conceptualizing an emergent collaborative organization. Journal of Social Entrepreneurship, v. 7, n. 1, p. $49-73,2016$.

TURKER, D.; VURAL, C. A. Embedding social innovation process into the institutional context: Voids or supports. Technological Forecasting and Social Change, v. 119, p. 98-113, 2017.

VAN DER HAVE, R. P.; RUBALCABA, L. Social innovation research: An emerging area of innovation studies? Research Policy, v. 45, n. 9, p. 19231935,2016

VAZQUEZ-MAGUIRRE, M.; PORTALES, L. Profits and purpose: Organizational tensions in social enterprises. Intangible Capital, v. 14, n. 4, p. $604-618,2018$.

WEERAWARDENA, J.; MORT, G. S. Competitive strategy in socially entrepreneurial nonprofit organizations: Innovation and differentiation. Journal of Public Policy \& Marketing, v. 31, n. 1, p. 91-101, 2012.

WITKAMP, M. J.; ROYAKKERS, L. M. M.; RAVEN, R. P. J. M. From cowboys to diplomats: Challenges for social entrepreneurship in the Netherlands. Voluntas: international journal of voluntary and nonprofit organizations, v. 22, n. 2, p. 283-310, 2011.

YU, X. Social Entrepreneurship in China's Non-profit Sector. China Perspectives, n. 3, p. 53, 2016

YUNUS, Muhammad. Social business entrepreneurs are the solution. In: The Future Makers. Routledge, 2017. p. 219-225.

ZEBRYTE, I.; JORQUERA, H. Chilean tourism sector "B Corporations": evidence of social entrepreneurship and innovation. International Journal of Entrepreneurial Behavior \& Research, v. 23, n. 6, p. 866-879, 2017. 